\title{
CONTROLE DE LA POPULATION DE CHIENS DANS LA MUNICIPALITE DE JOÃO PESSOA (BRÉSIL) PENDANT LES QUATRE ANS DE 2015 A 2018
}

\author{
Gabriella Menezes Freitas SILVA ${ }^{1}$; Gil Dutra FURTADO²; Bianca Miranda AMORIM³ ${ }^{3}$ José Andreey
} Almeida TELES ${ }^{4}$; Marcos Antônio Jerônimo COSTA ${ }^{5}$; Felipe Eduardo da Silva SOBRAL ${ }^{6}$

${ }^{1}$ Diplôme en Médecine Vétérinaire/Centro Universitário Maurício de Nassau, João Pessoa, Brésil (UNINASSAU). Email: gabriellamenezess@hotmail.com

${ }^{2}$ Diplôme en Médecine Vétérinaire/UNINASSAU; Agronome/Universidade Federal da Paraíba, Brésil (UFPB); Doctorat en Psychobiologie/Universidade Federal do Rio Grande do Norte, Brésil (UFRN); Ingénieur Agronome Partenaire de la Coopérative Agroalimentaire, Brésil (COOPAGRO). E-mail: gdfurtado@hotmail.com

${ }^{3}$ Diplôme en Médecine Vétérinaire/UNINASSAU. E-mail: biamorim1999@outlook.com

${ }^{4}$ Médecine Vétérinaire/Universidade Federal de Campina Grande, Brésil (UFCG); Spécialiste des Maladies InfectieusesContagieuses des Animaux Domestiques/Universidade Federal Rural de Pernambuco, Brésil (UFRPE); Master en Bioscience Animale/UFRPE; Docteur en Sciences Vétérinaires/UFRPE; Coordinateur et Conférencier du Cours de Médecine Vétérinaire/UNINASSAU.E-mail: teles.jaa@gmail.com

${ }^{5}$ Biologiste/UFPB; Doctorat en Sciences Biologiques/UFPB; Professeur/Universidade Estadual da Paraíba, Brésil (UEPB). E-mail: marcoscostajp@gmail.com

${ }^{6}$ Médecine Vétérinaire/UFCG; Master en Sciences Animales/UFCG; Professeur de Médecine Vétérinaire/UNINASSAU. E-mail: felipe_eduardo_med_vet@hotmail.com

La castration est un outil puissant dans la recherche de la qualité de vie des animaux domestiques, en particulier chez les animaux de compagnie pour cesser définitivement les modifications comportementales et hormonales liées aux fonctions physiologiques de la reproduction, en plus de contribuer directement pour la réduction de la dissémination des zoonoses par le contrôle de la population. Parmi les chiens, l'intervention chirurgicale chez les femelles, affecte directement la réduction du risque de développer des néoplasies, principalement des carcinomes mammaires et élimine la survenue d'infections utérines, déjà chez les mâles, cette procédure contribue à réduire la frustration les rapports sexuels par la réduction de la libido, ainsi que l'anxiété générée lors de la recherche de partenaires pour la reproduction, minimisent également l'incidence des problèmes de la prostate et empêchent le développement de carcinomes testiculaires. Le centre de surveillance de l'environnement et des zoonoses de la municipalité de João Pessoa (Brésil), en charge d'un rôle très important en ce sens, effectue des castrations de chiens et de chats en ville et à partir de ce travail, a été utilisé une méthodologie de recherche documentaire dans des documents spécifiques de l'organisme public et avec ceux-ci effectuant des évaluations statistiques dans les données collectées. Il a été constaté qu'entre 2015 et 2018, 1517 castrations ont été effectuées sur des chiens des deux sexes, avec une croissance de 177 animaux castrés en 2015 pour 660 animaux en 2018, et présentant une moyenne de 379 animaux par an, un résultat qui présente $0,05 \%$ de signification et démontre l'importance que cette action a eu pour la ville. L'action élective, avec l'action d'un médicament vétérinaire de prévention de l'intervention chirurgicale dans l'appareil reproducteur, montre une action bénéfique qui procure une action sanitaire souhaitée par la communauté en général. Il est conclu que la castration est méthodologiquement présentée comme une technique sûre pour améliorer le bien-être des animaux domestiques, tout en contribuant à la prévention de la multiplication indésirable des animaux domestiques et, par conséquent, à la dissémination des zoonoses.

Mots-clés: Animaux domestiques; Dissémination des zoonoses; Canins. 